\title{
ÍNDICES DE MORTANDAD LÉXICA EN PUERTO RICO: AFRONEGRISMOS
}

El rubro de "mortandad léxica" cobija a varios fenómenos que, aunque de efectos paralelos, presentan causas muy diversas. La pérdida total de una lengua o de parcelas de la misma puede ser resultado del empobrecimiento paulatino de la lengua materna en situaciones bilingües, cuando el contacto se da con una lengua más poderosa política y socialmente; suele ser el caso de las minorías étnicas en un mundo lingüístico ajeno, aunque no faltan casos de sustitución de lenguas en los que no se ha producido proceso migratorio alguno. El español de algunas comunidades de los Estados Unidos ${ }^{1}$, por una parte, y los muy conocidos casos del gaélico ${ }^{2}$ y del español de Trinidad ${ }^{3}$, por otra, ilustran ambas situaciones.

${ }^{1}$ Cf., por ejemplo, los trabajos aparecidos en los siguientes volúmenes colectivos: E. Hernández-Chávez, A. D. Cohen y A. F. Belgrano (eds.), El lenguaje de los chicanos, Center for Applied Linguistics, Arlington, VA, 1975; C. BowEN y J. ORNSTEIN (eds.), Studies in Southwest Spanish, Newberry House, Rowley, MA, 1976; G. Keller, R. Techner y S. Viera (eds.), Bilingualism in the Centennial and beyond, Bilingual Press, Jamaica-New York, 1976; R. DurÁN (ed.), Latino language and communicative behavior, Ablex Publishing, Norwood, NJ, 1981; J. Fishman y G. KeLler (eds.), Bilingual education for Hispanic students in the United States, Teacher's College Press, New York, 1982; y J. Amastae y L. Elías-Olivarfs (eds.), Spanish in the United States. Sociolinguistic aspects, Cambridge University Press, Cambridge, 1982.

2 Véanse, de N. C. Dorian, sus estudios "Grammatical change in a dying dialect", Lan, 49 (1973), 413-438; "Gender in a terminal dialect", Scottish Gaelic Studies, 7 (1976), parte 2; "The problem of the semi-speaker in language death", International Joumal of the Sociology of Language, 12 (1977), 23-32; "A hierarchy of morphophonimic decay in Scottish Gaelic language death: the differential failure of lenition", Word, 28 (1977), 96-109; "The fate of morphological complexity in language death: evidence from East Sutherland Gaelic", Lan, 54 (1978), 590609 ; y "Language lost and maintenance in language contact situations", comunicación presentada a la Language Skill Attrition Conference, University of Pennsylvania, 1985.

${ }^{3} \mathrm{~S}$. Moodie lo ha estudiado detenidamente: "The Spanish language as spo- 
La neurolingüística habla también de mortandad para referirse al estado de deterioro lingüístico en que suelen desembocar los afásicos, y, sin necesidad de que intervenga elemento patológico alguno, a la erosión del lenguaje que se observa en algunos hablantes de avanzada edad.

La lingüística aplicada a la enseñanza de lenguas extranjeras y la psicolingüística de la adquisición usan el término mortandad para designar la pérdida de destrezas lingüísticas en aquellos estudiantes que, habiendo estudiado una segunda lengua, han dejado de utilizarla ${ }^{4}$.

Por último, también se habla de mortandad cuando la competencia lingüística de una comunidad de habla reduce algunos de los inventarios que antes la integraban, sin que lo hayan motivado factores externos de carácter lingüístico.

La dialectología y la sociolingüística, sin embargo, han explorado poco este terreno. Aparentemente cerrado ya el efímero capítulo de la glotocronología - a pesar de la garbosa defensa de S. Gudschinsky ${ }^{5}$ - ninguna de las dos disciplinas ha mostrado entusiasmo por estas pesquisas. En este sentido las investigaciones de H. López Morales ${ }^{6}$, O. Alba ${ }^{7}$ y M. Vaquero ${ }^{8}$ sobre mortan-

ken in Trinidad", Caribbean Studies, 13 (1973), 88-94; "The phonemic system of the Spanish of Trinidad', Caribbean Studies, 13 (1973), 95-98; El español hablado en la isla de Trinidad, tesis doctoral inédita, Universidad Complutense de Madrid, 1980; "Trinidad Spanish pronouns: A case of language death in the Caribbean", en R. Nash y D. Belaval (eds.), Readings in Spanish-English contrastive linguistics, Inter-American University Press, Hato Rey, Puerto Rico, 1982, t. 3, pp. 206228; "El español de Trinidad: variabilidad y desgaste articulatorio", ALHis, 2 (1986), 177-195; "Examen del léxico de un dialecto obsolescente: el español de Trinidad", ACIEA (1), pp. 593-599; y "Morphophonemic illformedness in an obsolescent dialect: A case study of Trinidad Spanish", Orbis (en prensa). En sus últimos trabajos Moodie habla de dialecto obsolescente, siguiendo la ya clásica terminología de M. Swadesh, "Sociological notes on obsolescent languages", IJAL, 14 (1948), 226-235.

${ }^{4}$ Véase R. LAMBert y B. Freed (eds.), The loss of language skills, Newbury, Rowley, MA, 1982.

5 "The ABC's of lexicostatistics (glottochronology)", Word, 12 (1965), 175-210.

6 “'Indigenismos en el español de Cuba", en Estudios sobre el español de Cuba, Las Américas Publishing, New York, 1971, pp. 50-61; véase también de ese volumen "Elementos africanos en el español de Cuba", pp. 62-71.

7 "Indigenismos en el español hablado en Santiago [de los Caballeros, República Dominicana]", $A L M, 14$ (1976), 71-100.

8 "El léxico indígena en el español hablado en Puerto Rico" e "Índices sociolingüísticos de los indigenismos de Puerto Rico", ambos en su libro Léxico ma- 
dad léxica de indigenismos y afronegrismos en Las Antillas son trabajos aislados que no han producido descendencia ${ }^{9}$. Es cierto que hoy la dialectología hispanoamericana cuenta con estudios importantes sobre la norma léxica de diversas comunidades de habla, y que éstos permiten establecer contrastes con los inventarios recogidos en diccionarios y en otros repertorios lexicográficos, pero es evidente que el análisis de la mortandad, léxica en este caso, no constituye per se la meta que los guía ${ }^{10}$.

La influencia negra en el español de Puerto Rico ha sido estudiada con mucho detalle por M. Álvarez Nazario ${ }^{11}$. Aunque otros investigadores han abordado el tema -C. Mauleón, G. de Granda, W. W. Megenney ${ }^{12}-$ El elemento afronegroide en el español de Puerto Rico sigue siendo, particularmente en su segunda edición, el libro más acabado sobre el tema. En el aspecto léxico, el capítulo tercero recoge una nómina de 131 lexemas simples y compuestos (excluyo del recuento topónimos y antropónimos) que el autor considera de uso pasado o actual, especificando algunos casos en que no hay coincidencia semántica entre ambos. Los 131 afronegrismos que constituyen la base de la presente investigación (véase Apéndice A) aparecen divididos en siete campos léxicos ${ }^{13}$ :

rinero de Puerto Rico y otros estudios, Playor, Madrid, 1986, pp. 127-148 y 149-193.

${ }^{9}$ El libro de los profesores M. Sala, D. Munteanu, V. Neagu y T. Sandru-Olteanu, El léxico indígena del español americano. Apreciaciones sobre su vitalidad, Academia Mexicana-Editura Academeie Romane, México-Bucuresti, 1977, es un buen intento de ofrecer el estado de la cuestión. Han tenido que trabajar con los materiales disponibles en diccionarios y monografías dialectales, lo que significa que no han podido manejar datos estadísticos de frecuencia de uso. Cf. también M. SALA, "Sobre la vitalidad de los indigenismos americanos", BdFS, 31 (1980-81), 429-434, hecho sobre los mismos criterios.

${ }^{10}$ Sirva de ejemplo el trabajo pionero y excelente de JuAN M. LOPE BLANCH, El léxico indígena en el español de México, El Colegio de México, México, 1969.

${ }^{11}$ El elemento afronegroide en el español de Puerto Rico. Contribución al estudio del negro en América, Instituto de Cultura Puertorriqueña, San Juan, 1961. Utilizo la $2^{a}$ ed., publicada también por el Instituto de Cultura en 1974.

${ }^{12}$ C. C. Mauleón, El español de Loiza Aldea, Eds. Partenón, Madrid, 1974; G. DE GRANDA, "Algunos rasgos morfosintácticos de posible origen criollo en el habla de áreas hispanoamericanas de población negra", $A L M, 14$ (1976), 5-22; W. W. Megenney, "Sub-Saharan influences in lexicon of Puerto Rico", Orbis, 30 (1983), 214-260.

${ }^{13}$ Tomo directamente los lexemas que Álvarez Nazario da como afronegrismos sin entrar a examinar las etiologías propuestas, algunas de las cuales no están exentas de serios problemas, como es el caso del controvertible chévere; cf. la 
CuAdro 1

Campos léxicos estudiados

\begin{tabular}{lll}
\hline A & La flora & $001-023$ \\
$\mathrm{~B}$ & La fauna & $024-031$ \\
$\mathrm{C}$ & El individuo & $032-057$ \\
$\mathrm{D}$ & Vida material & $058-076$ \\
$\mathrm{E}$ & Vida espiritual & $077-118$ \\
$\mathrm{~F}$ & Vida en sociedad & $119-125$ \\
$\mathrm{G}$ & Léxico misceláneo & $126-131$ \\
\hline
\end{tabular}

Nuestro propósito es corroborar la vitalidad de esos lexemas y su pertenencia, por tanto, a la norma léxica actual de Puerto Rico.

Para ello se reunió una muestra de 256 sujetos procedentes de todo el país; en ella estaba integrada una submuestra de 36 informantes pertenecientes a dos núcleos demográficos fuertemente conformados por elementos negros étnicos y - se supone - culturales: Loíza Aldea y el barrio ponceño de San Antón ${ }^{14}$. Uno de nuestros objetivos era corroborar si existían diversos índices de vitalidad de afronegrismos entre ambas poblaciones.

El muestreo utilizado fue de carácter empírico con afijación uniforme; las variables que concurrieron en su diseño fueron sexo, nivel generacional y nivel sociocultural, establecido este último mediante una sumatoria ponderada de tres parámetros de base: escolarización, profesión e ingresos ${ }^{15}$. Se manejaron tres niveles

etiología pintoresca propuesta por J. J. ARrom, "Origen y semántica de la palabra chévere", 'HNBA, pp. 17-24. El profesor Álvarez Nazario ha tenido la amabilidad de informarme en comunicación personal que no cree ya en el étimo africano de este lexema. Otras observaciones menores en cuanto a etimologías expuestas en este libro pueden verse en la reseña de J. M. LOPE BLANCH en NRFH, 16 (1962), especialmente p. 455, nota 4. Tampoco altero su clasificación de lexemas en campos léxicos, aunque en algunos casos de bailes modernos (chachachá, mambo, samba) pudiera cuestionarse su inclusión dentro del campo de la vida espiritual.

${ }^{14}$ Intervinieron en la muestra sujetos de Aguadilla, Aibonito, Añasco, Arecibo, Bayamón, Caguas, Canóvanas, Carolina, Cataño, Cayey, Ceiba, Cidra, Coamo, Comerío, Fajardo, Guánica, Guayama, Guayanilla, Guaynabo, Juan Díaz, Las Marías, Las Piedras, Loíza, Luquillo, Manatí, Mayagüez, Naranjito, Patillas, Ponce, Sabana Grande, Salinas, San Germán, San Juan, San Lorenzo, Trujillo Alto, Vega Baja, Villalba y Yabucoa, es decir, 39 de los 78 municipios (un $50 \%$ ) en que está dividida la isla.

${ }^{15}$ Para detalles relativos a la elaboración del parámetro nivel socio-cultural, véase H. LÓPEZ-MORALES, Estratificación social del español de San Juan de Puerto Rico, UNAM, México, 1983, especialmente pp. 27-30. 
de edad $(20-34,35-54,55+)$ y dos estratos socioculturales, uno medio alto y otro bajo.

Aunque el trabajo de R. Andersen sobre "language attrition" va dirigido al estudio de otro tipo de mortandad lingüística (lenguas en contacto, retención de segundas lenguas) ${ }^{16}$ sus postulados metodológicos resultan aprovechables como red subyacente. Andersen señala que un estudio integral de la mortandad debe encargarse de analizar tanto la producción como la comprensión, lo relativo a la lengual oral y a la escrita, los diversos niveles de lengua (fonológico, gramatical, léxico), las diversas funciones del uso lingüístico (argumentar, quejarse, disculparse, etc.), los dominios del uso (casa, oficina, iglesia), las actividades comunicativas (un discurso, una conferencia, un informe técnico, una conversación informal...) y, por último, el nivel del discurso.

$\mathrm{El}$ instrumento preparado para recoger los materiales de nuestra investigación contemplaba tanto la producción como la comprensión, aunque se circunscribía al estudio del léxico y de la lengua oral. Tampoco se ocupaba de examinar los afronegrismos en contexto lingüístico, pero sí en contexto comunicativo, pues aunque no se siguió la especificación tripartita propuesta por Andersen (funciones, dominios y actividades) se trabajó con variación diafásica - estilo espontáneo $v$ s. estilo cuidadoso- lo que sin duda se sóbreimpone al patrón recomendado por el investigador norteamericano. Sin embargo, este último aspecto permanece aún sin analizar y, por lo tanto, no formará parte del presente estudio.

Una vez recopilados los datos se les sometió a tratamiento estadístico para obtener las frecuencias absolutas (FA) y relativas (FR) de todos los lexemas. A continuación el corpus léxico que examinamos fue dividido, de acuerdo con la frecuencia obtenida, en siete grupos: I (100), II (99.9-80), III (79.9-60), IV (59.9-40), V (39.9-20), VI (19.9-3) y VII (2.9-0) (véase Apéndice B).

Considerando que la norma léxica debería estar integrada por lexemas que hubiesen alcanzado una frecuencia de uso superior al $60 \%$, reunimos en uno solo los grupos I, II y III para constituir el de la norma léxica activa (NLA) de los afronegrismos, y dejamos intactos los otros cuatro para poder estudiar los grados de mortandad ${ }^{17}$.

${ }^{16}$ R. W. ANDERSEN, "Determining the linguistic attributes of language attrition", en R. Lambert y B. Freed (eds.), The loss of language skills, pp. 83-118.

${ }^{17}$ Estos cortes - como cualquier otro- son totalmente arbitrarios, pero el lector tiene la oportunidad de ir al Apéndice B y establecer sus propias delimitacio- 
El cuadro 2 muestra claramente que casi un $40 \%$ de todo este léxico pertenece al último de los grupos, y que dentro de éste son 24 los lexemas (un $48 \%$ ) que tienen frecuencia 0 . Si se suman las cifras correspondientes a la norma léxica activa, por una parte, y las de los restantes cuatro grupos, por otra, concluimos que la norma está constituida por el $26.7 \%$ frente al 73.2 que agrupa al vocabulario que se halla en proceso hacia la mortandad o que ha llegado ya a la etapa final.

Cuadro 2

Número de lexemas que integran los siete grupos de frecuencias

\begin{tabular}{rcrr}
\hline Grupo & & $N$ & $\%$ \\
\hline I & $(100)$ & 6 & 4.5 \\
II & $(99.9-80)$ & 7 & 5.3 \\
III & $(79.9-60)$ & 22 & 16.7 \\
IV & $(59.9-40)$ & 11 & 8.3 \\
V & $(39.9-20)$ & 14 & 10.6 \\
VI & $(19-9-3)$ & 21 & 16.0 \\
VII & $(2.9-0)$ & 50 & 38.1 \\
\hline
\end{tabular}

Cuando se analizan los campos léxicos de acuerdo con los grupos de frecuencia de uso obtenemos los siguientes datos:

CuADRo 3

Índices de vitalidad y mortandad por campos léxicos

\begin{tabular}{crccccc} 
& & & \multicolumn{5}{c}{ Mortandad } \\
\cline { 5 - 7 } $\begin{array}{c}\text { Campo } \\
\text { léxico }\end{array}$ & $N$ & $N L A$ & 1 & 2 & 3 & 4 \\
\hline A & 23 & 34.7 & 0 & 21.7 & 26.0 & 17.3 \\
B & 8 & 50.0 & 0 & 0 & 0 & 50.0 \\
C & 26 & 30.0 & 15.3 & 7.6 & 19.2 & 26.9 \\
D & 19 & 36.8 & 17.7 & 15.7 & 15.7 & 15.7 \\
E & 42 & 16.6 & 11.9 & 4.7 & 11.9 & 54.7 \\
F & 7 & 14.2 & 0 & 28.5 & 28.5 & 28.5 \\
G & 6 & 0 & 0 & 33.3 & 50.0 & 16.6 \\
\hline
\end{tabular}

nes. La validez que pueden tener es de índole práctica, puesto que facilitan la comparación con la norma léxica activa de los afronegrismos usados en Cuba; cf. H. López Morales, "Elementos africanos en el español de Cuba", que también parte del $60 \%$ de uso para el establecimiento de la norma. 
El índice más alto de NLA está en el campo de la fauna, seguido por el de la vida material, el de la flora y el del individuo. La mayor tasa de mortandad pertenece al léxico misceláneo $(100 \%)$, seguido por los de vida en sociedad $(85.5 \%)$, vida espiritual $(83.2 \%)$, el individuo $(70 \%)$, la flora $(65.3 \%)$, vida material $(62.8 \%)$ y la fauna $(50 \%)$.

Sólo al cementerio léxico constituido por los términos del grupo 4 pertenecen -entre otros- plátano fotoco y plátano guimbo, belembe (Xanthosoma brasiliense), calalú 'planta comestible'; chalungo 'chapucero', malungo 'gallo o gallina grande', macuenco 'flaco, enclenque, débil', matungo 'desmedrado, flacucho'; ñango 'individuo de miembros torcidos y débiles', cocolo 'negro esclavo procedente de las Antillas menores', macaco 'feo', mambi 'sospechoso de deslealtad al régimen español', mendé 'ciertas prácticas folklóricas', pian 'enfermedad de la piel', tengue 'estado de majadería infantil'; malambo 'clase de machete', fufú 'comida hecha a base de plátanos, calabazas, malanga o ñames hervidos y luego amasados', pon 'tarta de batata, calabaza y yautía majadas con harina de maíz y melaza'; bambulaé 'baile y son de bomba', leró 'baile de bomba', macandá 'brujería', grasimá 'baile de bomba'; bululú 'alboroto, tumulto, escándalo', yubá 'jolgorio, fiesta, diversión bulliciosa'; ñafitear 'sisar, hurtar'. Imagínese cuánto se abrirá el abanico de mortandad tan pronto como abandonamos este grupo 4, integrado por lexemas de frecuencias entre 2.9 y 0 , y examinamos los demás grupos de mayor frecuencia.

El análisis de la submuestra de 36 sujetos procedentes de Loíza Aldea y del barrio de San Antón, en Ponce, demuestra que la situación es casi paralela con respecto a la mortandad. No vive en estos informantes ninguno de los lexemas que el resto del país ha hecho desaparecer. Por un momento pensé que yuba' 'nombre de baile de bomba' era conocido por una de mis informantes de Loíza, pero pronto me di cuenta de que sólo recordaba una palabra del estribillo de una bomba ${ }^{18}$.

Eso sí, la citada submuestra alcanzó frecuencias de uso mayores en los lexemas bambulaé, cocobalé, ambos, tipos de bomba; fufú 'hacer brujo', baquiné 'canto cuando se muere un niño', mongo 'débil' y sambumbia 'sobras, comidas viejas'.

${ }^{18}$ Puse especial atención en la encuesta de Loíza en el lexema miñana 'cierta clase de abono', que resultaba totalmente desconocido en el resto del país, porque C. C. Mauleón lo recogió allí a principios de la década de los 70 , pero ninguno de mis informantes, ni aun los más viejos, lo conocían. Cf. Maulón, op. cit., p. 141. 
No se corrobora, pues, la hipótesis de que estas comunidades, más apegadas que el resto del país a tradiciones y costumbres de procedencia africana, mantengan vivos más lexemas de este origen. Las diferencias que se señalan entre ellas y otras zonas de Puerto Rico, si es que realmente existen, no son de orden léxico.

Los datos a los que he aludido anteriormente pertenecen todos a la competencia léxica activa de los hablantes. No es necesario que nos detengamos en muchos detalles explicativos para poder afirmar que el paso de esta competencia a la pasiva, integrada por términos que se comprenden pero que nunca se usan en la actuación lingüística, es un punto intermedio en el camino hacia la muerte léxica.

Aquellas unidades que figuran en las nóminas pasivas de la competencia (véase Apéndice $\mathrm{C}$ ) son muchas (83.9\%), pero alcanzan frecuencias relativamente bajas. Siguiendo el patrón estadístico anterior, he constituido un grupo - I-, que llamo norma léxica pasiva - NLP-, con aquellos lexemas que muestran una frecuencia superior al $60 \%$ sobre la cifra más alta alcanzada: a este grupo pertenecen los lexemas que obtuvieron frecuencias relativas entre 25 y 15 . Con el resto de la nómina se conformaron otros dos: II (14.9-6), III (5.9-0.3).

$\mathrm{Al}$ primer grupo pertenece el $10 \%$ del total del inventario pasivo; al segundo, el $38.1 \%$, y al tercero el $51.1 \%$, lo que parece demostrar que un porcentaje relativamente reducido es el que debe considerarse como norma léxica.

El cuadro 4 muestra el resultado de estos cómputos por campos léxicos:

Cuadro 4

Índices de léxico pasivo por campos léxicos

\begin{tabular}{ccccc}
\hline $\begin{array}{c}\text { Campo } \\
\text { léxico }\end{array}$ & $N$. & $1(N L P)$ & 2 & 3 \\
\hline A & 20 & 0 & 47.8 & 43.4 \\
B & 7 & 0 & 0 & 87.5 \\
C & 23 & 3.8 & 46.1 & 30.7 \\
D & 15 & 21.0 & 31.5 & 21.0 \\
E & 39 & 11.9 & 21.4 & 57.1 \\
F & 6 & 0 & 57.1 & 14.2 \\
G & 6 & 16.6 & 16.6 & 66.6 \\
\hline
\end{tabular}


En lo que a norma de léxico pasivo se refiere sólo son cuatro los campos léxicos afectados: la vida material, el léxico misceláneo, la vida espiritual y el individuo.

Las relaciones que pudieran existir entre la pasivización del léxico y los índices de mortandad se hacen evidentes tras el estudio de los datos que nos entrega el cuadro 5.

Cuadro 5

Indices de mortandad léxica y de vocabulario de uso pasivo (NLP), contrastado con el léxico vivo (NLA)

\begin{tabular}{cccc}
\hline $\begin{array}{c}\text { Campo } \\
\text { léxico }\end{array}$ & NLA & $\begin{array}{c}\text { Mortandad } \\
(1-4)\end{array}$ & $\begin{array}{c}\text { NLP } \\
(1)\end{array}$ \\
\hline A & 34.7 & 65.3 & 0 \\
B & 50.0 & 50.0 & 0 \\
C & 30.0 & 70.0 & 3.8 \\
D & 36.8 & 63.2 & 21.0 \\
E & 16.6 & 83.4 & 11.9 \\
F & 14.2 & 85.8 & 0 \\
G & 0 & 100.0 & 16.6 \\
\hline
\end{tabular}

La tasa de mortandad por campo léxico no ofrece demasiados paralelos con el de pasivización. El léxico misceláneo está todo él dentro del proceso de mortandad; el proceso seguirá por más tiempo puesto que aún hay un $16.6 \%$ en la etapa intermedia de pasivización. Más lento parece ser el del léxico relativo a la vida material y a la espiritual, ya que todavía quedan cifras importantes en la NLP. En el resto de los casos, los procesos parecen encontrarse en estadios más avanzados, con 0 o cifras muy cercanas a ella.

El análisis del proceso de mortandad, estudiado en tiempo aparente (en el sentido laboviano de la expresión) arroja resultados muy elocuentes. La tercera generación es la que ofrece mayor índice de conservación de afronegrismos, seguida de la segunda; es decir, que a medida que bajamos en el espectro generacional aumenta la mortandad. Sin embargo, las diferencias no son muy relevantes estadísticamente: $2.7 \%$ de la tercera a la segunda generación y 3.8 de ésta a la primera, para una diferencia entre las generaciones extremas de $6.5 \%$.

Parecidas cifras y proporciones presenta la conservación: $1.4 \%$ de la primera a la segunda generación, 3.9 de ésta a la tercera. 
El patrón de pasivización de lexemas es, sin embargo, irregular: la que más pasiviza es la segunda generación, la que menos, la tercera. A pesar de que el patrón aquí no es estratificacional como se esperaría, puede descubrirse que los jóvenes ya han completado el proceso hacia la mortandad léxica en muchas ocasiones, mientras que las otras dos generaciones tienen más unidades en esta etapa intermedia.

En general, los datos obtenidos con estos cortes generacionales nos hablan de una mortandad antigua, ocurrida hace más de 55 años. La escasa diferencia en la tasa de mortandad que exhiben las generaciones nos asegura que presenciamos sólo restos de un vigoroso proceso casi consumado ya en el primer cuarto de este siglo o inclusive antes.

Esta conclusión, señalada por la estadística, obliga a replantear la cuestión de la mortandad de los afronegrismos. Si la analizamos desde un punto de vista sincrónico, no parece tratarse de una parcela del vocabulario que desaparece de la competencia lingüística de unos hablantes (jóvenes y viejos) sino que estas unidades nunca llegaron a integrar el lexicón de esos hablantes; habían muerto antes de que ellos entraran en la escena lingüística.

Las otras dos variables sociales consideradas en el estudio - -sexo y nivel sociocultural- quedaron totalmente neutralizadas. No hay relación asociativa alguna entre ellas y el léxico estudiado. Sólo podría subrayarse que el nivel bajo presenta un estado ligeramente más avanzado del proceso, pues ha hecho desaparecer más lexemas y ha situado menos en la nómina pasiva, situación que contrasta con el sociolecto medio-alto, pero las diferencias carecen de valor significativo.

Aunque la mortandad de afronegrismos en Puerto Rico no es fenómeno reciente, como se ha visto, es una realidad en la comunicación actual. Hay que reconocer, sin embargo, que la ausencia de esos lexemas no parece ser un impedimento para la buena marcha de los procesos comunicativos. Y ello se debe a que, en su mayoría, se trata de léxico cuyos designata han ido desapareciendo de la realidad del país, o se han confundido con otros, perdiendo rasgos de especificidad. Así, por ejemplo, nadie come hoy pon ni baila la cadenciosa mariangola ni practica el mendé, ni distingue más de tres o cuatro tipos de plátanos ni reconoce los múltiples tipos de bailes de bomba.

Muchos de estos términos han muerto; otros se han rescatado, provistos de un nuevo sentido: cocolo, por ejemplo, que ya no es un negro proveniente de las Antillas menores, sino un aficio- 
nado a la música de salsa. La muerte léxica ha sido consecuencia directa de la muerte de las cosas.

En resumen, la mortandad de los afronegrismos en Puerto Rico y en otras zonas del Caribe hispánico es sólo un caso más de lo que Andersen llama "cosmetic attrition", mortandad que no conlleva pérdida de la competencia lingüística, que a su vez reduce la comunicación y restringe la transmisión de información. Tampoco conlleva estigmatización alguna hacia el hablante; esta mortandad carece de atributos negativos de carácter socio-afectivo.

Pero sólo cuando dispongamos del resultado de otros dos estudios ("Nuevos significados para viejos afronegrismos" y "Variación diafásica en el uso de afronegrismos") tendremos una perspectiva más diáfana del uso de los afronegrismos en Puerto Rico.

Humberto López Morales

Universidad de Puerto Rico

\section{APÉNDICE A}

\section{A. LA FLORA}

001 guineo

002 plátano forrongo

003 plátano congo

004 plátano chamaluco

005 plátano chumbo

006 plátano güimbo

007 plátano fotoco

008 plátano mafafo

009 plátano malango

010 plátano maricondo

011 gandul

012 gunda

013 malanga

014 name

015 quingombó

016 malagueta

017 marimbo

018 anamú

019 belembe

020 palo cachimbo

021 calalú

022 yerba cangá

023 yonyón

B. La fauna

$\begin{array}{ll}024 \text { chalungo } & 028 \text { guinea } \\ 025 \text { macuenco } & 029 \text { chango } \\ 026 \text { malungo } & 030 \text { gongolí } \\ 027 \text { matungo } & 031 \text { changa }\end{array}$


G. El individuo

032 angolo
033 cocolía
034 cocolo
035 congo
036 mandinga
037 mendé
038 mozambique
039 membe/bemba
040 ñango
041 ñoco
042 dengue
043 monga
044 pian
D. VIDA MATERIAL

058 calalú

059 fufú

060 funche

061 marifinga

062 mofongo

063 tostón

064 bombotó

065 pon

066 guarapo

067 mamplé

E. VIDA ESPIRITUAL
045 cocorioco

046 cocoroco

047 changa

048 chango

049 cheche

050 chévere

051 macaco

052 mambí

053 mongo

054 ñangotarse

055 ñeñeñé

056 sucusumuco

057 tengue

068 abombarse

069 sambumbia

070 mondongo

071 cachimbo/ -a

072 candungo

073 motete

074 chimba

075 malambo

076 miñana

077 baquiné
078 mendé
079 fufú
080 macandá
081 yacó
082 bomba
083 bongó
084 cua
085 marimba
086 marimbo
087 marímbula
088 timba
089 bomba
090 bomba
091 babú
092 bambulaé
093 belén
094 bembé

077 baquiné

078 mendé

079 fufú

080 macandá

082 bomba

084 cua

085 marimba

086 marimbo

087 marímbula

094 bembé
099 cunyá

100 curiquinque

101 danuá

102 grasimá

103 guateque

104 leró

105 mariandá

106 mariangola

107 masón

108 milonga

109 sicá

110 timbeque

111 yubá

112 balele

113 cangüí

114 conga

115 chachachá

116 mambo 
095 calindá

096 candungué

097 cocobalé

098 cuembé

E. VIDA EN SOCIEDAD

$$
\begin{aligned}
& 119 \text { taita } \\
& 120 \text { bachata } \\
& 121 \text { bululú } \\
& 122 \text { calalú }
\end{aligned}
$$

G. LÉXiCo MISCELÁNEO

126 burundanga

127 farufa

128 fuácata
117 merengue

118 samba

123 pachangá

124 titingó

125 yubá

129 jelengue

\begin{tabular}{|c|c|c|}
\hline & $F A$ & $F R$ \\
\hline \multicolumn{3}{|l|}{ GRUPO I } \\
\hline 050 chévere & 256 & 100.0 \\
\hline 011 gandul & 256 & 100.0 \\
\hline 028 guinea & 256 & 100.0 \\
\hline 001 guineo & 256 & 100.0 \\
\hline 014 ñame & 256 & 100.0 \\
\hline 063 tostón & 256 & 100.0 \\
\hline \multicolumn{3}{|l|}{ GRUPO II } \\
\hline 062 mofongo & 255 & 99.6 \\
\hline 013 malanga & 247 & 96.4 \\
\hline 043 monga & 245 & 95.7 \\
\hline 042 dengue & 243 & 94.9 \\
\hline 117 merengue & 240 & 93.7 \\
\hline 066 guarapo & 221 & 86.3 \\
\hline 015 quingombó & 217 & 84.3 \\
\hline \multicolumn{3}{|l|}{ GRUPO III } \\
\hline 054 ñangotarse & 200 & 78.1 \\
\hline 118 samba & 200 & 78.1 \\
\hline 115 chachachá & 194 & 75.9 \\
\hline 116 mambo & 194 & 75.9 \\
\hline
\end{tabular}

130 mamplé

131 ñafitear

APÉNDICE B 


\begin{tabular}{lll}
\hline & $F A$ & $F R$ \\
\hline 068 abombarse & 194 & 75.9 \\
018 anamú & 192 & 75.0 \\
083 bongó & 190 & 74.2 \\
060 funche & 184 & 73.0 \\
008 plátano mafafo & 181 & 70.7 \\
053 mongo & 178 & 69.5 \\
089 bomba & 175 & 68.3 \\
016 malagueta & 173 & 67.5 \\
033 cocolía & 172 & 67.1 \\
030 gongolí & 172 & 67.1 \\
073 motete & 168 & 65.6 \\
029 chango & 166 & 64.8 \\
031 changa & 162 & 63.2 \\
090 bomba & 161 & 62.8 \\
120 bachata & 160 & 62.5 \\
072 candungo & 160 & 62.5 \\
039 bembe & 155 & 60.5 \\
048 chango & 154 & 60.1
\end{tabular}

\section{GRUPO IV}

041 ñoco

047 changa

085 marimba

077 baquiné

53.5

114 conga

079 fufú

069 sambumbia

061 marifinga

48.8

049 cheche

088 timba

055 ñeñeñé

\section{GRUPO V}

082 bomba

123 pachanga

071 cachimbo, -a

124 titingó

017 marimbo

126 burundanga

086 marimbo

046 cocoroco 


\begin{tabular}{|c|c|c|}
\hline & $F A$ & $F R$ \\
\hline 012 gunda & 56 & 21.8 \\
\hline 064 bombotó & 54 & 21.0 \\
\hline \multicolumn{3}{|l|}{ GRUPO VI } \\
\hline 094 bembé & 44 & 17.1 \\
\hline 022 yerba cangá & 42 & 16.4 \\
\hline 056 sucusumuco & 41 & 16.0 \\
\hline 032 angolo & 38 & 14.8 \\
\hline 035 congo & 34 & 13.5 \\
\hline 036 mandinga & 32 & 12.2 \\
\hline 005 plátano chumbo & 26 & 10.1 \\
\hline 127 farufa & 17 & 6.6 \\
\hline 129 jelengue & 16 & 6.2 \\
\hline 087 marímbula & 16 & 6.2 \\
\hline 020 palo cachimbo & 16 & 6.2 \\
\hline 074 chimba & 14 & 5.4 \\
\hline 130 mamplé & 14 & 5.4 \\
\hline 081 yacó & 12 & 4.6 \\
\hline 067 mamplé & 11 & 4.2 \\
\hline 002 plátano forrongo & 11 & 4.2 \\
\hline 010 plátano maricondo & 11 & 4.2 \\
\hline 058 calalú & 10 & 3.9 \\
\hline 119 taita & 10 & 3.9 \\
\hline 122 calalú & 9 & 3.5 \\
\hline 023 yonyón & 8 & 3.1 \\
\hline
\end{tabular}

Grupo VII

075 malambo
121 bululú
113 changüí
040 ñango
007 plátano fotoco
059 fufú
006 plátano guimbo
092 bambulaé
104 leró
037 mendé
044 pian
080 macandá
131 ñafitear
057 tengue
112 balelé
019 belembe
045 cocorioco
024 chalungo

$\begin{array}{ll}2 & 0.7 \\ 2 & 0.7\end{array}$




\begin{tabular}{lll}
\hline & $F A$ & $F R$ \\
\hline 102 grasimá & 2 & 0.7 \\
026 malungo & 2 & 0.7 \\
109 sicá & 2 & 0.7 \\
021 calalú & 1 & 0.3 \\
097 cocobalé & 1 & 0.3 \\
098 cuembe & 1 & 0.3 \\
103 guateque & 1 & 0.3 \\
111 yubá & 1 & 0.3 \\
091 babú & 0 & 0 \\
093 belén & 0 & 0 \\
095 calindá & 0 & 0 \\
096 candungué & 0 & 0 \\
034 cocolo & 0 & 0 \\
084 cua & 0 & 0 \\
099 cunyá & 0 & 0 \\
100 curiquenque & 0 & 0 \\
101 danuá & 0 & 0 \\
051 macaco & 0 & 0 \\
025 macuenco & 0 & 0 \\
052 mambí & 0 & 0 \\
106 mariangala & 0 & 0 \\
105 mariandá & 0 & 0 \\
107 masón & 0 & 0 \\
027 matungo & 0 & 0 \\
078 mendé & 0 & 0 \\
108 milonga & 0 & 0 \\
076 miñana & 0 & 0 \\
070 mondongo & 0 & 0 \\
038 mozambique & 0 & 0 \\
065 pon & 0 & 0 \\
110 timbeque & 0 & 0 \\
125 yubá & 0 & 0 \\
& &
\end{tabular}

GRUPO I

071 cachimbo, -a

126 burundanga

077 baquiné

036 mandinga

061 marifinga

115 chachachá

$\begin{array}{ll}64 & 25.0 \\ 62 & 24.2 \\ 48 & 18.7 \\ 46 & 17.9 \\ 44 & 17.1 \\ 43 & 16.7\end{array}$




\begin{tabular}{lll}
\hline & $F A$ & $F R$ \\
\hline 069 sambumbia & 42 & 16.4 \\
088 timba & 42 & 16.4 \\
072 candungo & 41 & 16.0 \\
085 marimba & 40 & 15.6 \\
116 mambo & 39 & 15.2
\end{tabular}

GRUPo II

\begin{tabular}{llr}
016 malagueta & 38 & 14.8 \\
105 mariandá & 33 & 12.8 \\
123 pachangá & 33 & 12.8 \\
124 titingó & 33 & 12.8 \\
035 congo & 32 & 12.5 \\
055 ñeñené & 32 & 12.5 \\
032 angolo & 30 & 11.7 \\
094 bembé & 30 & 11.7 \\
079 fufú & 30 & 11.7 \\
017 marimbo & 30 & 11.7 \\
086 marimbo & 30 & 11.7 \\
022 yerba cangá & 30 & 11.7 \\
033 cocolía & 29 & 11.3 \\
060 funche & 29 & 11.3 \\
039 bembe & 28 & 10.9 \\
046 cocoroco & 28 & 10.9 \\
120 bachata & 27 & 10.5 \\
073 motete & 27 & 10.5 \\
118 samba & 27 & 10.5 \\
128 fuácata & 25 & 9.7 \\
041 ñoco & 25 & 9.7 \\
003 plátano congo & 25 & 9.7 \\
008 plátano mafafo & 25 & 9.7 \\
056 sucusumuco & 25 & 9.7 \\
012 gunda & 24 & 9.3 \\
018 anamú & 23 & 8.9 \\
114 conga & 23 & 8.9 \\
059 fufú & 23 & 8.9 \\
103 guateque & 23 & 8.9 \\
020 palo cachimbo & 23 & 8.9 \\
119 taita & 22 & 8.5 \\
049 cheche & 21 & 8.2 \\
068 abombarse & 18 & 7.0 \\
092 bambulaé & 18 & 7.0 \\
083 bongó & 18 & 7.0 \\
021 calalú & 18 & 7.0 \\
066 guarapo & 18 & 7.0 \\
064 bombotó & 17 & 6.6 \\
047 changa & 17 & 6.6 \\
& & \\
\hline & & 30
\end{tabular}




\begin{tabular}{lll}
\hline & $F A$ & $F R$ \\
\hline 009 plátano malango & 17 & 6.6 \\
005 plátano chumbo & 17 & 6.6 \\
054 ñangotarse & 16 & 6.2
\end{tabular}

GruPo III

058 calalú
074 chimba
031 changa
127 farufa
030 gongolí
029 chango
067 mamplé
098 cuembé
048 chango
040 ñango
097 cocobalé
106 mariangola
004 plátano chamaluco
057 tengue
082 bomba
113 changüí
129 jelengue
130 mamplé
053 mongo
089 bomba
090 bomba
022 calalú
096 candungué
013 malanga
087 marímbula
095 calindá
051 macaco
080 macandá
045 cocorioco
024 chalungo
010 plátano maricondo
019 belembe
002 plátano forrongo
006 plátano guimbo
112 balelé
084 cua
075 malambo
052 mambí
078 mendé
015 quingombó
081 yacó

$\begin{array}{ll}5 & 1.9\end{array}$




\begin{tabular}{lll}
\hline & $F A$ & $F R$ \\
\hline 023 yonyón & 4 & 1.5 \\
042 dengue & 4 & 1.5 \\
026 malungo & 4 & 1.5 \\
099 cunyá & 3 & 1.1 \\
104 leró & 3 & 1.1 \\
025 macuenco & 3 & 1.1 \\
035 mendé & 3 & 1.1 \\
109 sicá & 3 & 1.1 \\
100 curiquenque & 2 & 0.7 \\
110 timbeque & 2 & 0.7 \\
111 yubá & 2 & 0.7 \\
091 babú & 1 & 0.3 \\
121 bululú & 1 & 0.3 \\
102 grasimá & 1 & 0.3 \\
131 ñafitear & 1 & 0.3 \\
007 plátano fotoco & 1 & 0.3 \\
\hline
\end{tabular}

